\title{
Deep amplicon sequencing for culture- free prediction of susceptibility or resistance to 13 anti-tuberculous drugs
}

\author{
Agathe Jouet $^{1,12}$, Cyril Gaudin ${ }^{1,12}$, Nelly Badalato (1) ${ }^{1}$, Caroline Allix-Béguec ${ }^{1}$, \\ Stéphanie Duthoy ${ }^{1}$, Alice Ferré ${ }^{1}$, Maren Diels ${ }^{2}$, Yannick Laurent ${ }^{1}$, \\ Sandy Contreras ${ }^{1}$, Silke Feuerriegel ${ }^{3,4}$, Stefan Niemann ${ }^{3,4}$, Emmanuel André $^{5}$ \\ Michel K. Kaswa ${ }^{6}$, Elisa Tagliani (10 ${ }^{7}$, Andrea Cabibbe (10 ${ }^{7}$, Vanessa Mathys ${ }^{8}$, \\ Daniela Cirillo (1) ${ }^{7}$, Bouke C. de Jong (10) ${ }^{9}$, Leen Rigouts ${ }^{9,10}$ and Philip Supply (1) ${ }^{11}$
}

@ERSpublications

The novel Deeplex Myc-TB molecular assay shows a high degree of accuracy for extensive prediction of susceptibility and resistance to 13 anti-tuberculous drugs, directly achievable without culture, which may enable fast, tailored tuberculosis treatment https://bit.ly/3bAvcAt

Cite this article as: Jouet A, Gaudin C, Badalato N, et al. Deep amplicon sequencing for culture-free prediction of susceptibility or resistance to 13 anti-tuberculous drugs. Eur Respir J 2021; 57: 2002338 [https://doi.org/10.1183/13993003.02338-2020].

ABSTRACT Conventional molecular tests for detecting Mycobacterium tuberculosis complex (MTBC) drug resistance on clinical samples cover a limited set of mutations. Whole-genome sequencing (WGS) typically requires culture.

Here, we evaluated the Deeplex Myc-TB targeted deep-sequencing assay for prediction of resistance to 13 anti-tuberculous drugs/drug classes, directly applicable on sputum.

With MTBC DNA tests, the limit of detection was 100-1000 genome copies for fixed resistance mutations. Deeplex Myc-TB captured in silico $97.1-99.3 \%$ of resistance phenotypes correctly predicted by WGS from 3651 MTBC genomes. On 429 isolates, the assay predicted $92.2 \%$ of 2369 first- and secondline phenotypes, with a sensitivity of $95.3 \%$ and a specificity of $95.0 \%$ ( $95.4 \%$ and $97.1 \%$, respectively, when excluding ethionamide). 83 out of 102 (81.4\%) residual discrepancies with phenotypic results involved pyrazinamide, ethambutol and ethionamide, and low-level rifampicin or isoniazid resistance mutations, all notoriously prone to phenotypic testing variability. Only two out of 96 (2.1\%) resistance phenotypes undetected by Deeplex Myc-TB had known resistance-associated mutations by WGS analysis outside Deeplex Myc-TB targets. Phenotype predictions from Deeplex Myc-TB analysis directly on 109 sputa from a Djibouti survey matched those of MTBSeq/PhyResSE/Mykrobe, fed with WGS data from subsequent cultures, with a sensitivity of $93.5 / 98.5 / 93.1 \%$ and a specificity of $98.5 / 97.2 / 95.3 \%$, respectively. Most residual discordances involved gene deletions/indels and 3-12\% heteroresistant calls undetected by WGS analysis or natural pyrazinamide resistance of globally rare "Mycobacterium canettii" strains then unreported by Deeplex Myc-TB. On 1494 arduous sputa from a Democratic Republic of the Congo survey, 14902 out of 19422 (76.7\%) possible susceptible or resistance phenotypes could be predicted culture-free.

Deeplex Myc-TB may enable fast, tailored tuberculosis treatment.

This article has been revised according to the correction published in the November 2022 issue of the European Respiratory Journal.

This article has supplementary material available from erj.ersjournals.com

Received: 15 June 2020 | Accepted after revision: 3 Sept 2020

Copyright OERS 2021. This version is distributed under the terms of the Creative Commons Attribution Licence 4.0. 


\section{Introduction}

Important gaps remain for the diagnosis of drug-resistant tuberculosis (TB). Less than a third of the around 484000 multidrug (MDR)- or rifampicin-resistant TB incident cases estimated in 2018 were diagnosed and treated [1]. Phenotypic drug susceptibility testing (pDST) takes weeks of culture, while conventional molecular tests only identify common drug resistance mutations in a few gene targets [2].

Whole-genome sequencing (WGS) can effectively predict drug resistance or susceptibility of Mycobacterium tuberculosis complex (MTBC) strains [3-5]. However, its routine use for mycobacterial diagnosis typically requires primary culture [6]. Even if complex DNA enrichment procedures or thermoprotective DNA extraction are used, sequence coverage depths obtained by WGS directly on specimens often remain poor, even on samples with high bacterial loads [7,8]. This limits the sensitivity and/or the degree of confidence for detecting resistance mutations, especially when borne by minority populations (defining heteroresistance) that can be predictive of treatment failure [9].

Targeted, amplicon-based deep sequencing represents an attractive alternative [10]. Selective amplification of relevant gene regions prior to sequencing reduces both the amount of required DNA and the interference of unrelated DNA sequences (human or from microbial flora). Sequence coverage depths and multiplexing of samples per sequencing run can be substantially increased [11].

A previously described amplicon-based assay targets predefined high-confidence resistance variants in six $M$. tuberculosis genomic regions only $[11,12]$. In contrast, the targeted deep-sequencing assay called Deeplex Myc-TB (GenoScreen, Lille, France) targets full sequences (i.e. coding sequence plus part of promoter region) or (most) relevant regions of 18 MTBC drug resistance-associated genes, combined with genomic targets for mycobacterial species identification and MTBC strain genotyping. Included in the assay, a fully automated web application is used for rapid and user-friendly analysis and interpretation of the sequencing data, obtained from Illumina sequencers. Variant detection is comprehensive, including not only mutations known to be associated with resistance or susceptibility, but also as-yet uncharacterised mutations that can be confronted with drug susceptibility phenotypes when available. This assay has already been used in national and regional TB drug resistance surveys, including those under the supervision of the World Health Organization (WHO) [13-15], prior to its extensive evaluation.

Here, we describe for the first time the details of the assay and evaluate its performance based on data from more than 4000 isolates and 1600 clinical specimens.

\section{Methods}

\section{Limit of detection}

The limit of detection of Deeplex Myc-TB was evaluated using purified DNA from well-characterised MTBC strains of the WHO Special Programme for Research and Training in Tropical Diseases (TDR) collection (now included in the Belgian Culture Collection of Microorganisms (BCCM), Antwerp, Belgium) [16]. Serial dilutions were done after DNA quantification using the Qubit dsDNA HS Assay (Thermo Fisher, Waltham, MA, USA). A Mycobacterium intracellulare strain from BCCM was used for evaluation of the limit of detection for nontuberculous mycobacteria (NTM) identification. After Deeplex Myc-TB amplification as per manual instructions, amplicon libraries were prepared using the Nextera XT kit and sequenced with 150-bp paired-end reads using a MiSeq sequencer (Illumina, San Diego, CA, USA). For the subsequent stages, analyses were performed automatically using the integrated bioinformatics pipeline version 1.3 implemented in the Deeplex Myc-TB web application (note S1 in the supplementary material).

Affiliations: ${ }^{1}$ GenoScreen, Lille, France. ${ }^{2}$ BCCM/ITM, Mycobacteria Collection, Institute of Tropical Medicine, Antwerp, Belgium. ${ }^{3}$ Molecular and Experimental Mycobacteriology, Research Center Borstel, Borstel, Germany. ${ }^{4}$ German Center for Infection Research (DZIF), Partner Site Hamburg-Lübeck-Borstel, Borstel, Germany. ${ }^{5}$ Laboratory of Clinical Bacteriology and Mycology, Dept of Microbiology and Immunology, KU Leuven, Leuven, Belgium. ${ }^{6}$ National Tuberculosis Program, Kinshasa, Democratic Republic of the Congo. ${ }^{7}$ Emerging Bacterial Pathogens, IRCCS San Raffaele Scientific Institute, Milan, Italy. ${ }^{8}$ Unit Bacterial Diseases Service, Infectious Diseases in Humans, Sciensano, Brussels, Belgium. ${ }^{9}$ Mycobacteriology Unit, Dept of Biomedical Sciences, Institute of Tropical Medicine, Antwerp, Belgium. ${ }^{10}$ Dept of Biomedical Sciences, Antwerp University, Antwerp, Belgium. ${ }^{11}$ Université de Lille, CNRS, INSERM, CHU Lille, Institut Pasteur de Lille, U1019-UMR 8204-CIIL (Center for Infection and Immunity of Lille), Lille, France. ${ }^{12}$ These authors contributed equally to this work.

Correspondence: Philip Supply, Institut Pasteur de Lille, U1019-UMR 8204-CIIL (Center for Infection and Immunity of Lille), 1 Rue du Prof Calmette, 59000 Lille, France. E-mail: philip.supplydibl.cnrs.fr 
In silico analysis of drug resistance prediction versus WGS

The catalogue of resistance-associated variants included in Deeplex Myc-TB was compared with the resistance determinants and their associated resistance phenotypes found in both the training set of 2099 MTBC genomes and those retrieved in the validation set of 1552 MTBC genomes published in WALKER et al. [3].

Deeplex Myc-TB phenotype prediction versus WGS and phenotypic testing

Comparisons of Deeplex Myc-TB variant detection and phenotype predictions versus WGS and pDST were performed using a set of 429 reference isolates, including 213 collected by the Belgian National TB Reference Center (Sciensano) between 2007 and 2015 (MDR strains) and between March and October 2013 (non-MDR strains) and 216 from the WHO-TDR collection [16]. For the WHO-TDR collection, pDST was performed by using the proportion method (on Löwenstein-Jensen or Middlebrook 7H11 agar medium for first- or second-line drugs, respectively) with critical concentrations of $0.2,40,4,2,2,6,10$ and $10 \mu \mathrm{g} \cdot \mathrm{mL}^{-1}$ isoniazid, rifampicin, streptomycin, ethambutol, ofloxacin, kanamycin, capreomycin and ethionamide, respectively, as described by VINCENT et al. [16]. For the Sciensano strain set, pDST was routinely done using the BACTEC MGIT960 system and TB-eXiST application (Becton Dickinson, Franklin Lakes, NJ, USA) for first-line drugs as per the manufacturer's instructions, and the radiometric BACTEC 460 TB system (Becton Dickinson) for second-line drugs including amikacin, ofloxacin, moxifloxacin, ethionamide and linezolid, as described in PFyfFer et al. [17] or CAMBAU et al. [18] for isolates before or after 2012, respectively. Deeplex Myc-TB testing was used as described earlier. WGS was performed at GenoScreen using the Nextera XT kit and 150-bp paired-end reads on a HiSeq 2500 sequencer (Illumina) in Rapid Run mode. WGS analysis was performed using a Bowtie2-based pipeline with an initial threshold of $85 \%$ allele frequency for variant calling, with subsequent search for minority variants under low-frequency detection mode, using at least one read in both forward and reverse directions, $5 \times$ read coverage, and a minimal Phred score of 30 to call an allele with a minimal frequency of $3 \%$. To avoid probable errors due to mislabelling of samples, Deeplex Myc-TB- and WGS-based phylogenetic lineage and spoligotype identifications were compared for consistency, and any isolate with more than three discordances between predicted and observed phenotypes was excluded from the analysis, as done elsewhere [3].

\section{Deeplex Myc-TB on clinical specimens}

Deeplex Myc-TB sequencing data from 109 sputum samples and WGS data from cultured isolates from the Djibouti survey were obtained as described in TAgLiani et al. [13]. Phenotype prediction from WGS data was performed by MTBSeq version 1.0.2 [19], PhyResSE version 1.0 with resistance-associated variant database version 29 [20] and Mykrobe version 0.8.1 [21].

Deeplex Myc-TB sequencing data of 1494 sputum samples from the Democratic Republic of the Congo (DRC) survey were obtained as described in КАYомо et al. [22]. Briefly, after Ziehl-Neelsen staining and standard smear microscopy grading of acid-fast bacilli (AFB), sputa were stored and transported in $96 \%$ ethanol at a ratio of 1:1 at room temperature. DNA was extracted using a Maxwell 16 Low Elution Volume DNA Purification System (Promega, Madison, WI, USA), and analysed with Deeplex Myc-TB kits using NextSeq and MiSeq platforms.

\section{Data availability}

Deeplex Myc-TB sequence reads were deposited in the National Center for Biotechnology Information Sequence Read Archive (SRA) under BioProject numbers PRJNA649788 (TDR/Sciensano), PRJNA633444 (Djibouti), PRJNA643157 and PRJNA643242 (DRC), PRJNA633380 (NTM), and PRJNA633106 (limit of detection analysis). WGS SRAs are available under BioProject numbers PRJEB31023 for the TDR collection, PRJNA393924 for the Djibouti dataset and PRJEB25999 for the Sciensano collection. A detailed description of datasets used in this study is available in supplementary table S1.

\section{Results}

Assay design, limit of detection and mycobacterial species identification

The major gene targets associated with resistance to 13 first- and second-line anti-tuberculous drugs/drug classes and the databases implemented in the web application are shown in figure 1, and tables 1 and 2 . Further details on the assay design are provided in note S2 in the supplementary material, including the additional targets amplified in the single 24-plex PCR for mycobacterial species identification, spoligotyping/single nucleotide polymorphism (SNP) typing and an internal control, and the analysis with the web application.

As Deeplex Myc-TB does not depend on a specific DNA extraction method, the assay's limit of detection was estimated as the fraction of detectable sequence variants in four replicated analyses using serially 


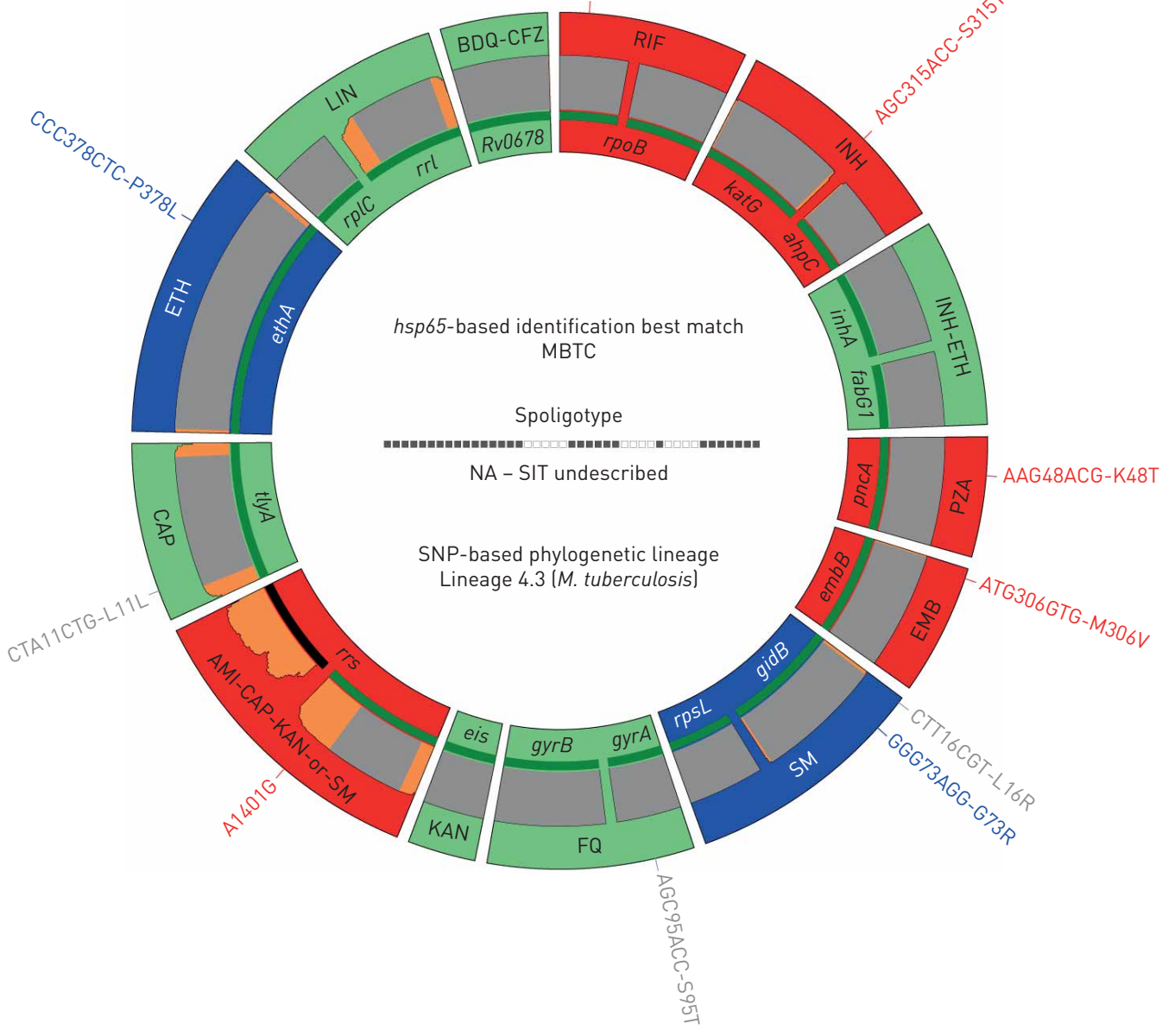

FIGURE 1 Deeplex Myc-TB results identifying a pre-extensively drug-resistant Mycobacterium tuberculosis complex (MTBC) strain in a sputum DNA sample collected in a tuberculosis drug resistance survey conducted in the Democratic Republic of the Congo. RIF: rifampicin; INH: isoniazid; PZA: pyrazinamide; EMB: ethambutol; SM: streptomycin; FQ: fluoroquinolones; KAN: kanamycin; AMI: amikacin; CAP: capreomycin; ETH: ethionamide; LIN: linezolid; BDQ: bedaquiline; CFZ: clofazimine; NA: not applicable; SIT: spoligotype international type; SNP: single nucleotide polymorphism; LOD: limit of detection. Information on hsp65 bestmatch-based identification, spoligotype (in this case, not yet known to the SITVIT database) and phylogenetic SNP-based identification of MTBC lineage is shown in the centre of circle. Information on drug susceptibility and drug resistance predictions for 13 anti-tuberculous drugs/drug classes is as follows. Target gene regions are grouped within sectors in a circular map according to the anti-tuberculous drug resistance with which they are associated. Sectors in red and green indicate targets in which resistance-associated mutations or either no mutation or only mutations not associated with resistance (shown in grey) are detected, resulting in predictions of resistant or susceptible phenotypes, respectively. Blue sectors refer to regions where as-yet uncharacterised mutations are detected. Green lines above/below gene names represent the reference sequences with coverage breadth $>95 \%$. LOD of heteroresistance (reflected by subpopulations of reads bearing a mutation) depends on the read depth at mutation position and is shown either as grey (LOD 3\%) or orange zones ( $L O D>3-80 \%$ ) above/below reference sequences. Here, LOD is $>3 \%$ at the end of a few targets only and over two rrs regions with usual lower coverage.

diluted purified, pre-quantified genomic DNA from three well-characterised MTBC strains and a mixture of two strains at a 5:95\% ratio. All (near-)fixed resistance variants were detectable with $10^{4}$ and $10^{3}$ genomes, and $83.3 \%$ with $10^{2}$ genomes (complete variant profiles obtained for 13 out of 16 tests) (figure 2). For resistance variants at $5 \%$ frequency, these fractions were $100 \%, 81.3 \%$ (with complete minor variant profiles in three out of four tests) and $43.8 \%$ (none with a complete minor variant profile) with 
TABLE 1 Mycobacterial/Mycobacterium tuberculosis complex (MTBC) genes or gene regions targeted by Deeplex Myc-TB

\begin{tabular}{lccc} 
Target & Genome position & Gene position & Codons \\
\hline rpoB1 & $760957-761355$ & $1151-1549$ & $384-517$ \\
rpoB2 & $760280-760812$ & $474-1006$ & $158-336$ \\
inhA & $1674287-1674880$ & $86-679$ & $29-227$ \\
fabG1 & $1673321-1673755$ & $-119-316$ & NA-106 \\
katG & $2155858-2155140$ & $254-972$ & $85-324$ \\
ahpC & $2726030-2726585$ & $-163-393$ & NA-131 \\
pncA & $2289301-2288672$ & $-60-+9$ & Full CDS \\
embB & $4247376-4248065$ & $863-1552$ & $288-518$ \\
gidB & $4408185-4407411$ & $18-+117$ & $6-N A$ \\
rpsL & $781536-781979$ & $-24-+45$ & Full CDS \\
rrs1 & $1472561-1473417$ & $716-1572$ & NA \\
rrs2 & $1471848-1472524$ & $3-679$ & NA \\
eis & $2715528-2715171$ & $-196-162$ & NA-54 \\
tlyA & $1917811-1918750$ & $-129-+4$ & Full CDS \\
gyrA & $7377-7754$ & $76-453$ & $26-151$ \\
gyrB & $6298-6943$ & $1059-1704$ & $353-568$ \\
ethA & $-9-+53$ & Full CDS \\
rplC & rrl & $300-+21$ & $100-N A$ \\
Rv0678 & $827482-4325951$ & $2266-2968$ & NA \\
hsp65 & $1475923-1476625$ & $-14-+52$ & Full CDS \\
\hline CDS & $778976-779539$ & $165-565$ & $55-189$
\end{tabular}

CDS: coding sequence; NA: not applicable for codons (positions outside a CDS, or in rrs or rrl rDNA regions). Positions of the reference sequences relative to the genome and genes of the M. tuberculosis H37Rv strain are indicated. Genome positions are indicated according to forward or reverse orientations of corresponding genes. For gene positions, minus $(-)$ or plus $(+)$ signs indicate positions in promoter or $3^{\prime}$ regions relative to the +1 or last nucleotide of CDSs, respectively.

$10^{4}, 10^{3}$ and $10^{2}$ genomes, respectively. Fixed and minority variants were not detected with $10^{1}$ genomes only (for limit of detection for MTBC and NTM identification, see supplementary figure S1 and S2, and note $\mathrm{S} 1$ in the supplementary material).

Out of 370 strains from 73 different NTM species/species complexes tested using DNA extracted from culture, 292 strains were identified at (sub)species or species complex levels by both reference and Deeplex Myc-TB testing. Of these, 274 (93.5\%) were correctly identified at (sub)species or species complex level by Deeplex Myc-TB (supplementary figure S3 and supplementary table S2, and note S7 in the supplementary material for methods and details). The 18 (6.2\%) strains that had taxonomically discordant results even at the complex level between both methods mostly consist of single discordant cases among otherwise

TABLE 2 Databases implemented in the Deeplex Myc-TB web application for mycobacterial species identification, Mycobacterium tuberculosis complex (MTBC) genotyping, and drug susceptibility and drug resistance prediction

\begin{tabular}{lcc} 
Database use & Database name & Reference \\
\hline Species identification & hsp65 & {$[23]$} \\
Spoligotyping & SITVITWEB & {$[24]$} \\
Lineage identification & Coll & {$[25]$} \\
& PhyResSE & {$[20]$} \\
Drug resistance prediction $^{\#}$ & Walker & {$[3]$} \\
& Miotto & {$[26]$} \\
& PhyResSE & {$[20]$} \\
& Walker & {$[3]$} \\
\end{tabular}

\footnotetext{
\#: priority is given to the collaborative, curated database ReSeqTB when detected variants are known to this database.
} 

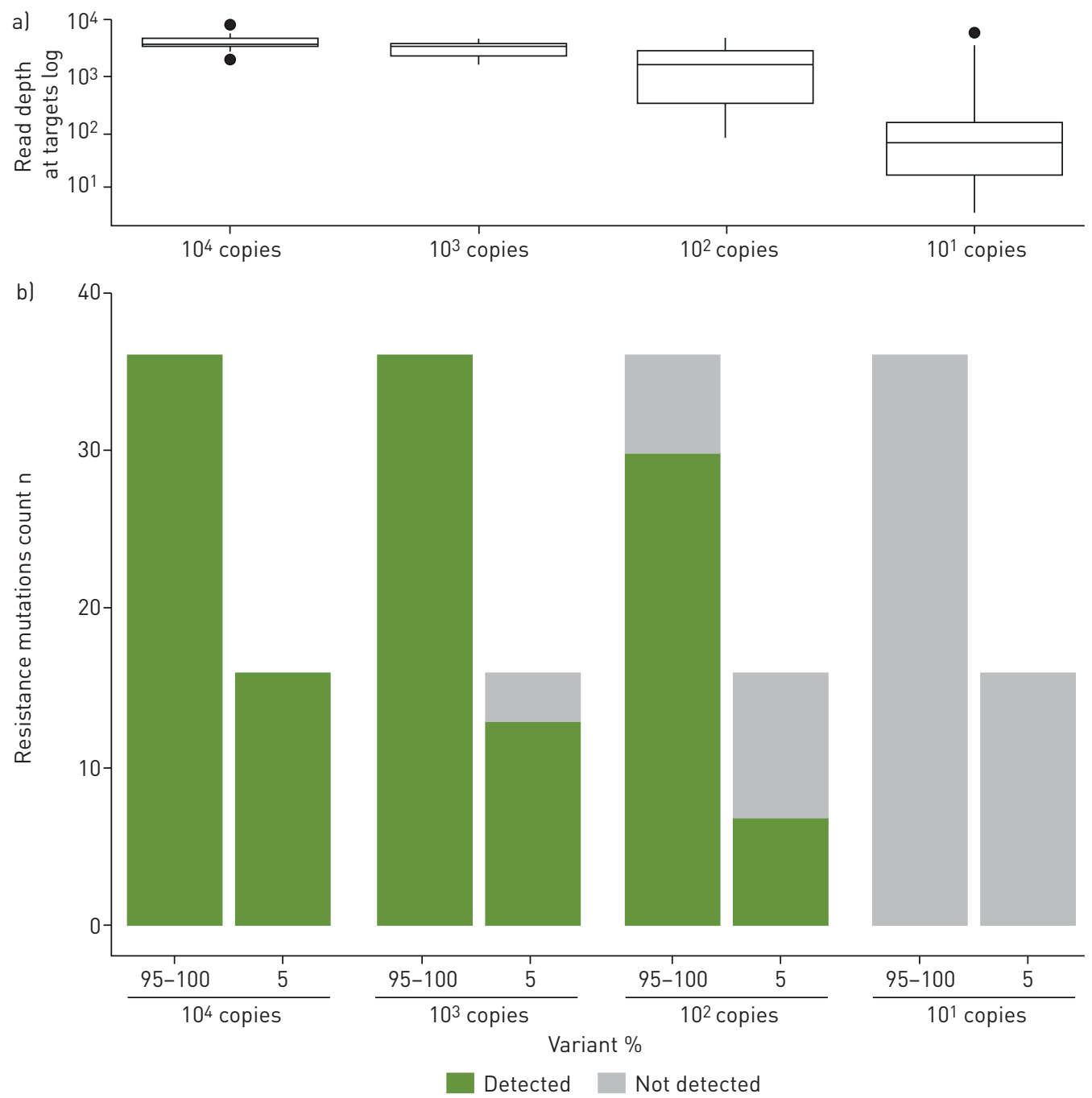

FIGURE 2 Limit of detection (LOD) of Deeplex Myc-TB for resistance variant detection. a) Read depth at resistance-associated Deeplex Myc-TB targets versus the number of input genomes. Box and whisker plots show median with interquartile range (IQR) and minimum-maximum range with a maximum of 1.5 IQR, respectively; outliers are indicated. b) For each dilution level with $10^{1}, 10^{2}, 10^{3}$ and $10^{4}$ genome copies, LOD was measured as the fraction of detected or undetected resistance variants in total sets of 36 (near-)fixed (95-100\% frequency) and 16 minority (5\% frequency) mutations, spread across four independent replicated tests of four different Mycobacterium tuberculosis complex genomic DNA extracts.

partially/fully concordant strains for a species (e.g. Mycobacterium ulcerans ( $\mathrm{n}=1 / 13)$ and Mycobacterium kansasii $(\mathrm{n}=1 / 14))$ or species rarely involved in infections (e.g. Mycobacterium peregrinum $(\mathrm{n}=2)$ ). However, for some of these few individual isolates of otherwise well-identified species, the correctness of the Deeplex Myc-TB identification was actually often possible or probable, as conflicting or ambiguous identifications were seen between the $r p o B$ and $16 \mathrm{~S}$ rDNA reference probes, with one or the other partially or fully matching the Deeplex Myc-TB result (e.g. M. kansasii versus Mycobacterium gastri) (supplementary table S2). The same often held true for the 16 residual isolates with discrepant subspecies within a matching complex (e.g. M. intracellulare versus Mycobacterium chimaera).

\section{Resistance variant detection versus WGS in silico}

We evaluated in silico the ability of Deeplex Myc-TB to capture 120 anti-tuberculous drug resistance-determining mutations spread across 14 genes, along with their concurrent first- and second-line resistance phenotypes, algorithmically identified in a WGS study by WALKER et al. [3]. Of these 120 resistance determinants, $106(88.3 \%)$ are included in the Deeplex Myc-TB targets and variant catalogue (supplementary table S3), spread across 13 of the 14 genes, the exception being $r p s A$, a minor target associated with pyrazinamide resistance. With these 106 variants, 644 out of the $663(97.1 \%)$ 
resistant phenotypes predicted by WGS in the WALKER et al. [3] training set of 2099 MTBC isolates were retrieved. Likewise, 53 out of the 58 resistance-determining mutations from the training set that were retrieved in the WALKER et al. [3] validation set of 1552 isolates were captured by Deeplex Myc-TB, enabling the prediction of 1199 out of 1207 (99.3\%) concurrent resistant phenotypes in this dataset (supplementary table S4).

\section{Phenotype prediction on isolates versus WGS and phenotypic testing}

We compared the ability of both Deeplex Myc-TB and Illumina-based WGS analysis to detect variants in DNA extracts from 429 MTBC strains. Of the 2403 variants identified in the Deeplex Myc-TB targets by either method, 2373 (98.8\%; 2293 SNPs and all 80 indels), including 797 (99.9\%) resistance variants, were detected by both Deeplex Myc-TB and our WGS pipeline under low-frequency mode (validated for accurate SNP calling in accordance with recent guidelines [28]). The remaining 30 (1.2\%) SNPs were all minority variants mostly with frequencies of $\sim 3-10 \%$ only identified by targeted deep sequencing, including one mutation associated with resistance (to ethambutol; note S4 in the supplementary material and supplementary table S5).

Deeplex Myc-TB drug susceptibility predictions based on these 2403 variants were compared with pDST results. In this set, 268 isolates were phenotypically resistant to at least one drug, including 156 MDR and six extensively drug-resistant isolates, resulting in 696 resistant and 1558 susceptible phenotypes. Of these 2254 phenotypes, 2080 (92.3\%) were predicted by Deeplex Myc-TB with a mean sensitivity of 95.2\% and a mean specificity of $95.0 \%$ (table 3 and supplementary table S6). The remaining 174 phenotypes (7.7\%) could not be predicted due to the presence of mutations uncharacterised in the variant database. When results were stratified by type of phenotypic method used as a reference, the concordance with genotypic predictions was slightly higher for phenotypes tested by liquid culture (supplementary table S7) compared with those tested by solid culture (supplementary table S8), for the three drugs principally assayed with both types of methods (rifampicin, isoniazid and ethambutol).

The proportion of resistant phenotypes accurately predicted as resistant by Deeplex Myc-TB was $>90 \%$ ( $90.7 \%$ for streptomycin to $100 \%$ for amikacin) for most individual drugs, except for pyrazinamide (85.7\%) and kanamycin (88.9\%, but reflecting nine isolates only) (table 3). For the MDR-defining compounds rifampicin and isoniazid, 159 out of 160 (99.4\%) and 176 out of 179 (98.3\%) isolates with resistance variants were correctly classified as resistant, accounting for $98.1 \%$ and $94.1 \%$ of all rifampicinand isoniazid-resistant isolates, respectively. For pyrazinamide, although all (42 out of $42(100 \%)$ ) isolates containing resistance variants for this drug were correctly predicted as resistant, these accounted for only $79.2 \%$ of all isolates with phenotypic resistance to pyrazinamide. Of note, seven minority resistance variants (with frequencies of 21.1-77\%) successfully predicted resistance to pyrazinamide $(n=3)$, fluoroquinolones, kanamycin, capreomycin and ethionamide ( $\mathrm{n}=1$ each).

Of the 630 resistant phenotypes with Deeplex Myc-TB predictions (i.e. not uncharacterised), only 30 $(4.8 \%)$ were predicted as susceptible due the absence of resistance-associated mutation in the Deeplex Myc-TB targets. In these phenotypes, the possible presence of high-confidence resistance-associated mutations was searched in extended regions (full coding sequence and promoter regions) of genes from which only (most) critical regions are covered by Deeplex Myc-TB, as well in 14 other, secondary resistance-associated gene targets, outside the Deeplex Myc-TB target regions, such as the embA promoter region (including, e.g. the $\mathrm{C}-12 \mathrm{~T}$ and $\mathrm{C}-16 \mathrm{~T}$ mutations) or the extended eth $A$ promoter region (including, e.g. the T-11C mutation) (supplementary table S9). WGS analysis detected a high-confidence resistance mutation in these regions for only one of these phenotypes (ethionamide resistant; L203L in fabG1 [4]) (supplementary table S9). Likewise, fabG1 L203L was the sole established resistance-associated mutation (for an isoniazid-resistant phenotype) detected by WGS outside of the assay's targets in 66 phenotypes uncharacterised by Deeplex Myc-TB.

Of the 1450 susceptible phenotypes with prediction, only $72(5.0 \%)$ were discordantly predicted as resistant. They all involved ethambutol and $e m b B$ mutations, ethionamide and eth $A$ frameshift-causing indels (mechanistically expected to cause ethionamide resistance [30]), or known low-level isoniazid/ ethionamide (inhA S94A, ahpC G-48A, fabG1 C-15T), rifampicin ( $r p o B$ L452P, H445N and D435Y) or streptomycin ( $g i d B \mathrm{~A} 138 \mathrm{~V})$ resistance mutations, all notoriously associated with poor phenotypic reproducibility (note S4 in the supplementary material) [3, 27, 31-33]. When excluding ethionamide, which is especially prone to such phenotypic testing errors, the overall sensitivity of Deeplex Myc-TB phenotype predictions was $95.4 \%$ and the overall specificity was $97.1 \%$, when uncharacterised mutations were not considered (table 3 ).

As acquisition of resistance to isoniazid is generally the first step towards drug resistance [34], a predicted susceptibility to isoniazid could a contrario be considered to predict susceptibility to other first-line drugs, 
TABLE 3 Deeplex Myc-TB phenotype predictions versus phenotypic drug susceptibility testing (pDST) on 429 reference isolates from the World Health Organization TDR and the Belgian National TB Reference Center (Sciensano) collections

\begin{tabular}{|c|c|c|c|c|c|c|c|c|c|c|c|c|c|c|}
\hline \multicolumn{5}{|c|}{ Phenotypically resistant } & \multicolumn{5}{|c|}{ Phenotypically susceptible } & \multicolumn{2}{|c|}{ All } & \multicolumn{2}{|c|}{ Excluding uncharacterised } & \multirow{3}{*}{$\begin{array}{c}\text { Uncharacterised } \\
\%\end{array}$} \\
\hline \multicolumn{4}{|c|}{ Deeplex Myc-TB n } & \multirow{2}{*}{$\begin{array}{c}\text { Total } \\
\mathbf{n}\end{array}$} & \multicolumn{4}{|c|}{ Deeplex Myc-TB n } & \multirow{2}{*}{$\begin{array}{c}\text { Total } \\
\mathbf{n}\end{array}$} & \multirow{2}{*}{$\begin{array}{c}\text { Sensitivity } \\
\%\end{array}$} & \multirow{2}{*}{$\begin{array}{c}\text { Specificity } \\
\%\end{array}$} & \multirow{2}{*}{$\begin{array}{c}\text { Sensitivity } \\
\%\end{array}$} & \multirow{2}{*}{$\begin{array}{c}\text { Specificity } \\
\%\end{array}$} & \\
\hline $\mathbf{R}$ & $\mathbf{R}_{\mathbf{h}}$ & $\mathbf{s}$ & U & & $\mathbf{R}$ & $\mathbf{R}_{\mathbf{h}}$ & $\mathbf{S}$ & $\mathbf{U}$ & & & & & & \\
\hline 159 & 0 & 1 & 2 & 162 & 3 & 0 & 241 & 6 & 250 & 98.1 (94.7-99.4) & $98.8(96.5-99.6)$ & $99.4(96.5-99.9)$ & $98.8(96.4-99.6)$ & 1.9 \\
\hline 176 & 0 & 3 & 8 & 187 & 3 & 0 & 190 & 32 & 225 & $94.1(89.8-96.7)$ & $98.7(96.2-99.5)$ & $98.3(95.2-100)$ & $98.4(95.5-99.5)$ & 9.7 \\
\hline 39 & 3 & 7 & 4 & 53 & 0 & 0 & 135 & 4 & 139 & $79.2(66.5-88.0)$ & $100(97.3-100)$ & $85.7(73.3-92.9)$ & $100(97.2-100)$ & 4.2 \\
\hline 95 & 0 & 8 & 5 & 108 & 26 & 2 & 273 & 3 & 304 & $88.0(80.5-92.8)$ & $90.8(87.0-93.6)$ & $92.2(85.4-96.0)$ & $90.7(86.9-93.5)$ & 1.9 \\
\hline 49 & 0 & 5 & 36 & 90 & 1 & 0 & 91 & 33 & 125 & $54.4(44.2-64.3)$ & $99.2(95.6-99.9)$ & $90.7(80.1-96.0)$ & $98.9(94.1-99.8)$ & 32.1 \\
\hline 21 & 1 & 2 & 3 & 27 & 1 & 0 & 120 & 4 & 125 & 81.5 (63.3-91.8) & $99.2(95.6-99.9)$ & 91.7 (74.2-97.7) & $99.2(95.5-99.9)$ & 4.6 \\
\hline 9 & 0 & 0 & 0 & 9 & 0 & 0 & 54 & 2 & 56 & $100(70.1-100)$ & $100(93.6-100)$ & $100(70.1-100)$ & $100(93.4-100)$ & 3.1 \\
\hline 7 & 1 & 1 & 0 & 9 & 0 & 0 & 72 & 5 & 77 & $88.9(56.5-98.0)$ & $100(95.2-100)$ & $88.9(56.5-98.0)$ & $100(94.9-100)$ & 5.8 \\
\hline 14 & 1 & 1 & 2 & 18 & 3 & 0 & 112 & 4 & 119 & $83.3(60.8-95.6)$ & $97.5(92.8-99.1)$ & $93.8(71.7-98.9)$ & $97.4(92.6-99.1)$ & 4.4 \\
\hline 24 & 1 & 2 & 6 & 33 & 33 & 0 & 70 & 15 & 118 & $75.8(59.0-87.2)$ & $72.0(63.3-79.3)$ & $92.6(76.6-97.9)$ & $68.0(58.4-76.2)$ & 13.9 \\
\hline 0 & 0 & 0 & 0 & 0 & 0 & 0 & 20 & 0 & 20 & NA & $100(83.9-100)$ & NA & $100(83.9-100)$ & 0 \\
\hline 593 & 7 & 30 & 66 & 696 & 70 & 2 & 1378 & 108 & 1558 & $86.2(83.4-88.6)$ & $95.4(94.2-96.3)$ & $95.2(93.3-96.6)$ & $95.0(93.8-96.0)$ & 7.7 \\
\hline 569 & 6 & 28 & 60 & 663 & 37 & 2 & 1308 & 93 & 1440 & $86.7(83.9-89.1)$ & $97.3(96.5-98.1)$ & $95.4(93.4-96.8)$ & $97.1(96.1-97.9)$ & 7.3 \\
\hline
\end{tabular}
Data for sensitivity and specificy isolates with this data available. The unit of analysis was therefore a phenotype, not an isolate. Results for bedaquiline/clofazimine are not shown, as there was no pDST comparator. R: detection of at least one fixed resistance-associated mutation; $R_{\mathrm{h}}$ : detection of resistance due to a minority variant (heteroresistance); S: detection of mutations known not to be associated with resistance (phylogenetic, benign, synonymous) or no mutation detected; U: detection of at least one nonsynonymous uncharacterised mutation in the absence of a resistance-associated mutation; NA: not applicable; WGS: whole-genome sequencing. " : a beta version of the Deeplex Myc-TB kit used for this analysis did not yet include the $a h p C$ promoter region including two isoniazid resistance-associated mutations G-48A and C-57T (note S2 in the supplementary material). We checked for these two mutations in the WGS dataset and found only two samples (TB-TDR0013 and TB-TDR-0160) with a fixed G-48A mutation. They were considered in this comparison to reflect results as obtained with the up-to-date version containing this region, as Deeplex Myc-TB captured 100\% of the variants detected by WGS in the initial ahpC gene part and all other gene targets in this dataset (see text). " : a simulation of the inclusion of additional pyrazinamide resistance-associated mutations published in YADoN et al. [29] (not incorporated in the ReSeqTB and Deeplex Myc-TB databases) resulted in identification of only three additional resistance mutations, classified as uncharacterised by Deeplex Myc-TB. However, out of these three mutations, two were found in pyrazinamide-susceptible phenotypes and only one in a pyrazinamide-resistant phenotype. ${ }^{+}$: fluoroquinolones include ofloxacin and moxifloxacin 
for which gene targets have no drug resistance mutations but contain uncharacterised mutations, as shown for WGS-based analysis [4]. When doing so for Deeplex Myc-TB predictions, the diagnostic performance could be further improved, with proportions of uncharacterised phenotypes reduced to $0.5-2.6 \%$ for rifampicin, ethambutol and pyrazinamide, at the cost of a single incorrect prediction of a susceptible phenotype for each of these drugs (note S5 in the supplementary material and supplementary table S10). This conditional interpretation is left to the user's decision and not implemented in the Deeplex Myc-TB analysis algorithm.

\section{Deeplex Myc-TB on clinical specimens}

We compared variant detection and phenotype predictions based on available Deeplex Myc-TB sequencing data directly obtained from 109 clinical specimens from a nationwide survey conducted in Djibouti versus analysis of WGS data obtained after culturing [13]. Overall, 693 out of 752 total variants (92.2\%) were detected by both Deeplex Myc-TB and our WGS pipeline used under low-frequency detection mode. Of 59 (7.8\%) discordances, all but one (a fixed pncA SNP not detected by Deeplex Myc-TB, albeit on a well-covered position) were minority variants undetected by this WGS pipeline, consistent with the average coverage depth of 3921× at Deeplex targets by Deeplex Myc-TB versus 57× by WGS (note $\mathrm{S} 6$ in the supplementary material and supplementary table S11).

Deeplex Myc-TB phenotype predictions were matched to those obtained with WGS data independently analysed with the WGS-based analysis tools PhyResSE [20], Mykrobe [21] and MTBSeq [19]. In contrast to PhyResSE and Mykrobe, MTBSeq requires bioinformatic skills for local implementation and use. However, MTBSeq has a more complete resistance mutation panel [19] and was therefore used as a primary reference in the comparison.

With the 1150 predicted phenotypes by Deeplex Myc-TB (155 resistant and 995 susceptible), the mean sensitivity and specificity versus available MTBSeq predictions was $93.5 \%$ and $98.5 \%$, respectively (table 4 ). 108 additional phenotypes (8.6\%) were not predicted by Deeplex Myc-TB due to the presence of uncharacterised mutations. Agreement on resistance prediction was $100 \%$ for all applicable drugs, except for rifampicin (93.5\%) and pyrazinamide (71.4\%). The rifampicin score resulted from probable differences between primary samples directly tested by Deeplex Myc-TB and cultures used for WGS analysis. Indeed, two resistance predictions by MTBSeq (and also by PhyResSE and Mykrobe) (figure 3) reflected detection of two minor resistance-associated variants in $r p o B$ that were undetected by Deeplex Myc-TB despite high coverage depths at these positions, suggesting genotypic heterogeneity/contamination that was introduced/ amplified after sputum processing, during subsequent culturing or WGS (note S7 in the supplementary material). For pyrazinamide, the lower sensitivity of Deeplex Myc-TB versus MTBSeq mostly resulted from different interpretation of some sequence variants or different regions interrogated in " $M$. canettii" isolates, which are naturally resistant to pyrazinamide and locally prevalent in and highly restricted to Djibouti [35]. Deeplex Myc-TB did identify seven M. canettii-containing samples based on the phylogenetic SNP pncA A46A, but did not (then) explicitly predict resistance to pyrazinamide, in contrast to MTBSeq based on panD M117T (and Mykrobe, based on pncA A46A) (figure 3 and note S8 in supplementary material).

Conversely, 15 out of the 995 (1.5\%) phenotypes predicted as susceptible by MTBSeq (excluding uncharacterised phenotypes by Deeplex Myc-TB) were identified as resistant by Deeplex Myc-TB (table 4). Of these, $10(66.6 \%)$ discordances were due to minority resistance-associated variants at $3.3-12.8 \%$ only detected in sputa by Deeplex Myc-TB (figure 3 and note S7 in the supplementary material). Importantly, eight out of these 10 minority variants co-occurred with one or more minority resistance and/or phylogenetic variants within an individual sputum. This further supports true-positive variant calls reflecting genuine mixed strains and/or combined heteroresistance detected by targeted deep sequencing, missed by WGS analysis due to lower coverage depth or potential culture bias. The five remaining discordant resistance predictions resulted from divergent variant interpretation, involving one eth $A$ frameshift-causing indel (mechanistically expected to cause ethionamide resistance, see earlier), two debated $e m b B$ mutations and a gidB mutation $(n=2)$ associated with low-level streptomycin resistance [18] (note S7 in the supplementary material), which were associated with resistance by both Deeplex Myc-TB and PhyResSE and/or Mykrobe, but not by MTBSeq (figure 3).

The mean sensitivity and specificity of the phenotypes predicted by Deeplex Myc-TB versus available PhyResSE/Mykrobe predictions was $98.5 / 93.1 \%$ and $97.2 / 95.3 \%$, respectively (supplementary tables S12 and S13, and note S8 in the supplementary material,).

Although patient sputa were (presumably) all smear-positive as per study design, the rate of successful Deeplex Myc-TB sequence analysis versus microscopic grade was not investigated in the Djibouti survey. This parameter was therefore evaluated on a set of 1494 direct sputum samples from a nationwide survey conducted in the DRC [22]. Yield of culture from cetylpyridinium chloride-stored sputum largely suffered 
TABLE 4 Phenotype predictions by direct Deeplex Myc-TB analysis of DNA from 109 clinical specimens versus phenotype predictions by MTBSeq with whole-genome sequencing (WGS) data from culture

\begin{tabular}{|c|c|c|c|c|c|c|c|c|c|c|c|c|c|c|c|}
\hline & \multicolumn{5}{|c|}{ MTBSeq resistant } & \multicolumn{5}{|c|}{ MTBSeq susceptible } & \multicolumn{2}{|c|}{ All } & \multicolumn{2}{|c|}{ Excluding uncharacterised } & \multirow{3}{*}{$\begin{array}{c}\text { Uncharacterised } \\
\%\end{array}$} \\
\hline & \multicolumn{4}{|c|}{ Deeplex Myc-TB n } & \multirow{2}{*}{$\begin{array}{c}\text { Total } \\
\mathbf{n}\end{array}$} & \multicolumn{4}{|c|}{ Deeplex Myc-TB n } & \multirow{2}{*}{$\begin{array}{c}\text { Total } \\
\mathbf{n}\end{array}$} & \multirow{2}{*}{$\begin{array}{c}\text { Sensitivity } \\
\%\end{array}$} & \multirow{2}{*}{$\begin{array}{c}\text { Specificity } \\
\%\end{array}$} & \multirow{2}{*}{$\begin{array}{c}\text { Sensitivity } \\
\%\end{array}$} & \multirow{2}{*}{$\begin{array}{c}\text { Specificity } \\
\%\end{array}$} & \\
\hline & $\mathbf{R}$ & $\mathbf{R}_{\mathbf{h}}$ & $\mathbf{s}$ & $\mathbf{U}$ & & $\mathbf{R}$ & $\mathbf{R}_{\mathbf{h}}$ & $\mathbf{S}$ & U & & & & & & \\
\hline Rifampicin & 29 & 0 & 2 & 1 & 32 & 0 & 0 & 75 & 2 & 77 & $90.6(75.8-96.8)$ & $100(95.2-100)$ & $93.5(79.3-98.2)$ & $100(95.1-100)$ & 2.8 \\
\hline Isoniazid & 31 & 0 & 0 & 0 & 31 & 0 & 2 & 71 & 5 & 78 & 100 (89.0-100) & 97.4 (91.1-99.3) & 100 (89.0-100) & $97.3(90.5-99.2)$ & 4.6 \\
\hline Pyrazinamide & 18 & 2 & 8 & 3 & 31 & 0 & 3 & 73 & 1 & 77 & $64.5(46.9-78.9)$ & $96.1(89.2-98.7)$ & $71.4(52.9-84.7)$ & $96.1(89.0-98.6)$ & 3.7 \\
\hline Ethambutol & 21 & 1 & 0 & 1 & 23 & 2 & 2 & 79 & 3 & 86 & $95.7(79.0-99.2)$ & $95.3(88.6-98.2)$ & $100(85.1-100)$ & $95.2(88.3-98.1)$ & 3.7 \\
\hline Streptomycin & 14 & 0 & 0 & 0 & 14 & 2 & 2 & 35 & 23 & 62 & $100(78.5-100)$ & $93.5(84.6-97.5)$ & $100(78.5-100)$ & $89.7(76.4-95.9)$ & 30.3 \\
\hline Fluoroquinolones & 0 & 0 & 0 & 0 & 0 & 0 & 1 & 104 & 4 & 109 & NA & 99.1 (95.0-99.8) & NA & 99 (94.8-99.8) & 3.7 \\
\hline Amikacin & 5 & 0 & 0 & 0 & 5 & 0 & 0 & 67 & 1 & 68 & $100(56.6-100)$ & $100(94.6-100)$ & $100(56.6-100)$ & $100(94.6-100)$ & 1.4 \\
\hline Kanamycin & 5 & 0 & 0 & 0 & 5 & 0 & 0 & 66 & 2 & 68 & $100(56.6-100)$ & $100(94.6-100)$ & $100(56.6-100)$ & 100 (94.5-100) & 2.7 \\
\hline Capreomycin & 12 & 0 & 0 & 0 & 12 & 0 & 0 & 59 & 4 & 63 & $100(75.8-100)$ & $100(94.2-100)$ & $100(75.8-100)$ & $100(93.9-100)$ & 5.3 \\
\hline Ethionamide & 7 & 0 & 0 & 4 & 11 & 1 & 0 & 66 & 31 & 98 & $63.6(35.4-84.8)$ & $99.0(94.4-98.2)$ & $100(64.6-100)$ & 98.5 (92.0-99.7) & 32.1 \\
\hline Linezolid & 0 & 0 & 0 & 0 & 0 & 0 & 0 & 87 & 3 & 90 & NA & $100(95.9-100)$ & NA & $100(95.8-100)$ & 3.3 \\
\hline Bedaquiline & 0 & 0 & 0 & 0 & 0 & 0 & 0 & 99 & 10 & 109 & NA & $100(96.6-100)$ & NA & $100(96.3-100)$ & 9.2 \\
\hline Clofazimine & 0 & 0 & 0 & 0 & 0 & 0 & 0 & 99 & 10 & 109 & NA & $100(96.6-100)$ & NA & $100(96.3-100)$ & 9.2 \\
\hline Total & 142 & 3 & 10 & 9 & 164 & 5 & 10 & 980 & 99 & 1094 & 88.4 (82.6-92.5) & $98.6(97.7-99.2)$ & 93.5 (88.5-96.5) & $98.5(97.5-99.1)$ & 8.7 \\
\hline
\end{tabular}

Data for sensitivity and specificity are presented as mean (95\% CI): total sensitivity and specificity data are weighted means. Deeplex Myc-TB sequencing and WGS data originate from a report of a national tuberculosis drug resistance survey conducted in Djibouti [13]. Deeplex Myc-TB predictions were compared with WGS-based MTBSeq predictions separately for each drug, across isolates with this data available. R: detection of at least one fixed resistance-associated mutation; $R_{h}$ : detection of resistance due to a minority variant (heteroresistance); $\mathrm{S}$ : detection of mutations known not to be associated with resistance (phylogenetic, benign, synonymous) or no mutation detected; U: detection of at least one nonsynonymous uncharacterised mutation in the absence of a resistance-associated mutation; NA: not applicable. 


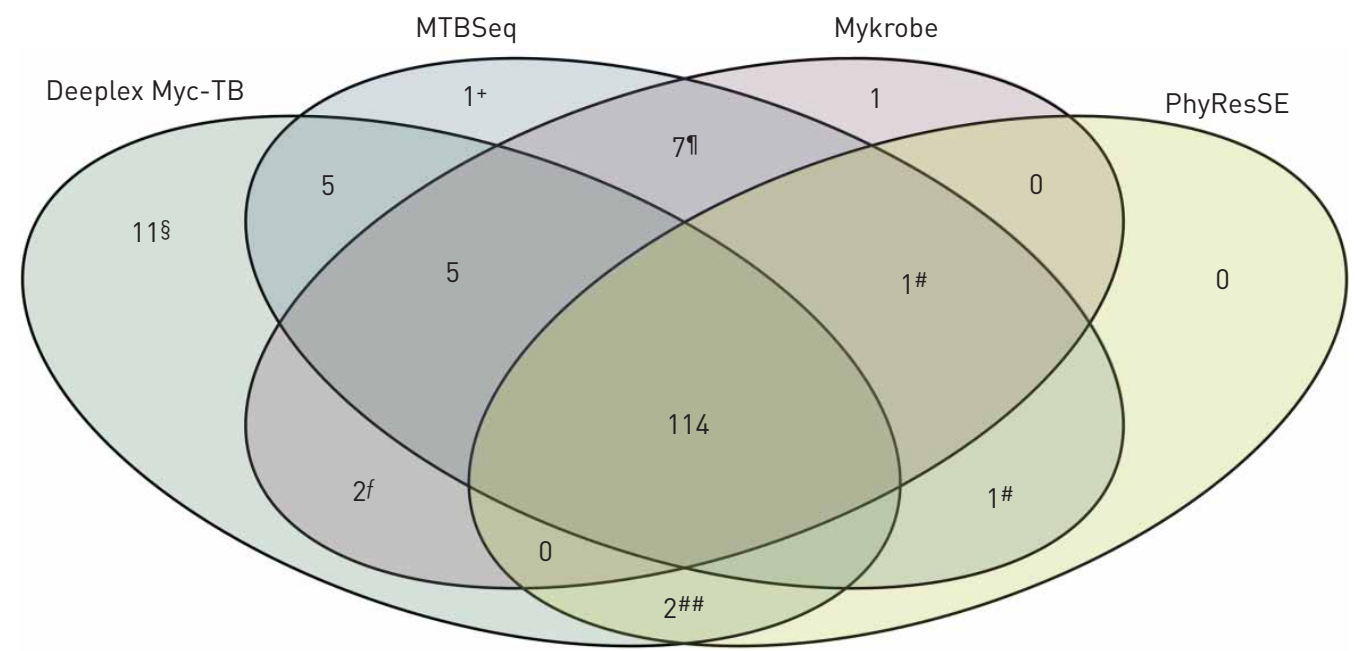

FIGURE 3 Venn diagram representing the agreement between resistant phenotypes identified by four Mycobacterium tuberculosis resistance and susceptibility prediction tools: Deeplex Myc-TB, MTBSeq, Mykrobe and PhyResSE. WGS: whole-genome sequencing. The numbers of resistant phenotypes predicted by Deeplex Myc-TB analysis on 109 sputum samples from Djibouti and other analysis tools fed with WGS data from corresponding cultures are shown. " : two rifampicin resistance phenotypes predicted by MTBSeq and PhyResSE and/or Mykrobe based on rpoB S431T and D435V, reflecting probable WGS or culture contaminations (see text); ": seven pyrazinamide resistance phenotypes predicted for "Mycobacterium canettii"-containing cultures by MTBSeq and Mykrobe based on panD M117T and pncA A46A, respectively; ${ }^{+}$: one pyrazinamide resistance phenotype predicted by MTBSeq based on pncA D136G; $\S$ : 11 resistant phenotypes predicted by Deeplex Myc-TB based on 10 minority variants (3-12\%) and one ethA frameshift-causing indel; $f_{\text {: }}$ two streptomycin resistance phenotypes predicted by Deeplex Myc-TB and Mykrobe based on gidB G69D; \#\#: two ethambutol resistance phenotypes predicted by Deeplex Myc-TB and PhyResSE based on embB S297A and Y319S.

from transport delays, whereas ethanol-preserved sputum samples were kept at room temperature for subsequent DNA extraction using a modified Maxwell 16 Low Elution Volume DNA Purification System [22]. Therefore, culture-free Deeplex Myc-TB testing was used mostly as a stand-alone assay for extensive pDST in TB patients, after their inclusion based on Ziehl-Neelsen smear positivity and positive M. tuberculosis detection on Xpert MTB/RIF.

Of the 1143 sputum samples with available microscopic examination data, mean pan-target read depth exceeded $1000 \times$ for samples graded $1+, 2+$ and $3+$, although, as expected, the dispersion towards lower values was inversely correlated with microscopic grading (figure $4 \mathrm{a}$ and b, and supplementary table S14). Broadly similar read depths were also observed for samples that were graded negative ( $\mathrm{n}=16$ only, as per the standard survey design normally enrolling smear-positive TB patients only) or without reported grading $(n=351)$. Despite the indeterminate bacterial loads in a third of the 1494 samples, MTBC was identified in $1258(84.2 \%)$ of them. Of the 19422 expected phenotypes in the 1494 samples, $73.5 \%$ (14277) were automatically predicted $(80.7-82.4 \%$ for the four first-line drugs), based on detected resistance mutations or absence of both resistance and uncharacterised mutations with minimal $5 \times$ read depth over $\geqslant 95 \%$ of the reference targets. Uncharacterised mutations were detected for an additional $5.9 \%$ $(\mathrm{n}=1137)$ and 625 additional susceptible phenotypes (3.2\%) could be predicted after verification of effective minimal coverage of all resistance positions in the corresponding target. When distinguished by microscopic grade, the proportion of predicted phenotypes in samples without microscopy results $(81.1 \%)$ was actually higher than for samples that were graded 0 (67.8\%), $1+(69.4 \%), 2+(69.5 \%)$ and $3+(76.3 \%)$.

Phenotypes for aminoglycosides, and to a lower extent linezolid, were relatively less frequently predicted, as a reflection of comparatively lower average read depths on $r r s$ and $r r l$ rDNA targets (supplementary table S14), expectedly due to competing commensal rDNA reads. However, such competition does not detectably affect specific calling of variants in samples with well-covered targets, as seen from the high degree of concordance with variants detected by WGS analysis on culture in the Djibouti dataset (except for minority variants only detected by deep sequencing that are likely true in most cases; note S6 in the supplementary material), and from full agreement between predictions of susceptible phenotypes for aminoglycosides and linezolid with those made by WGS analysis in the same dataset (table 4). 

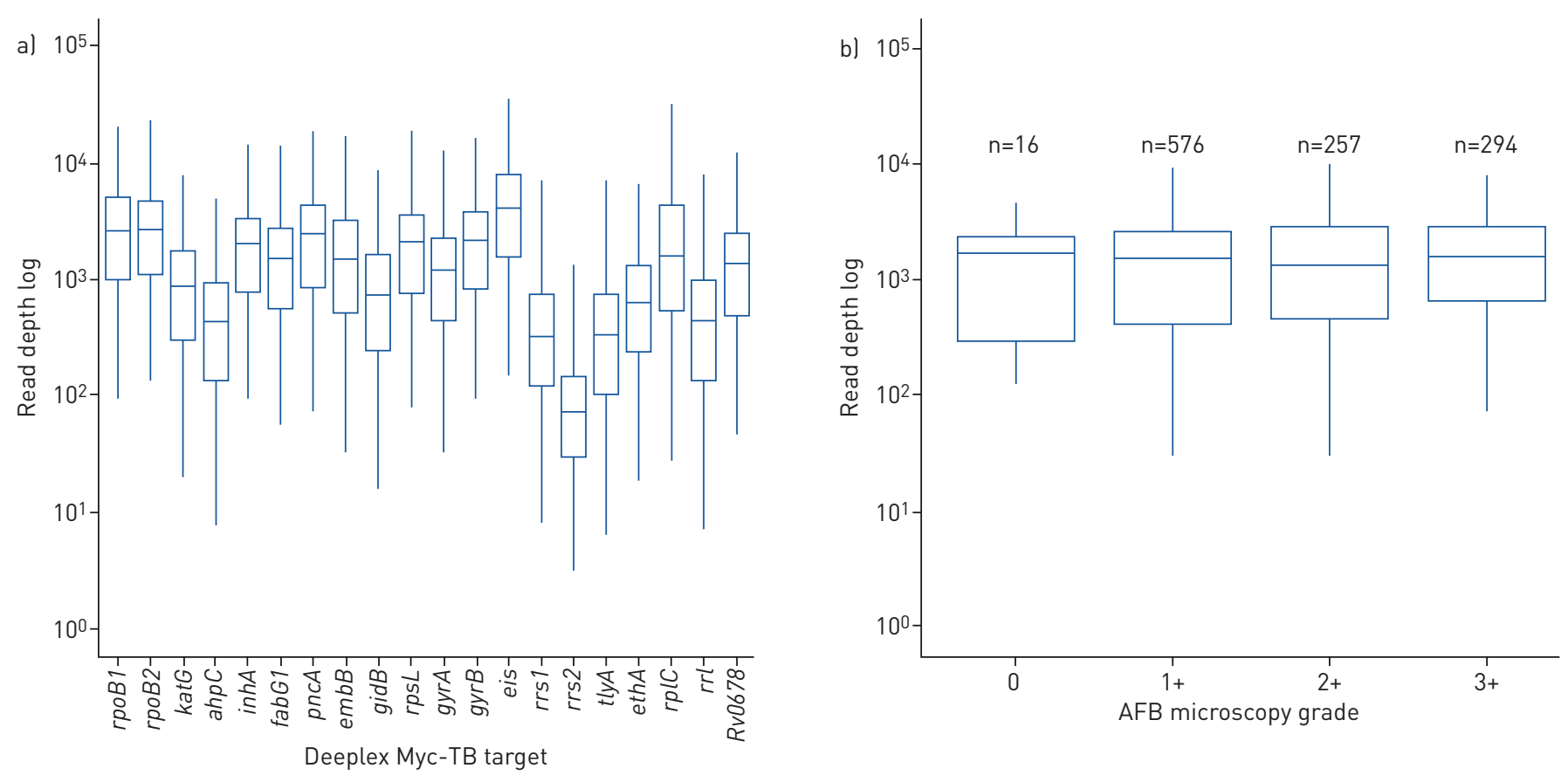

FIGURE 4 Log read depth obtained by direct Deeplex Myc-TB testing of DNA extracted from clinical specimens collected in a tuberculosis drug resistance survey conducted in the Democratic Republic of the Congo: a) log read depth at each drug resistance-associated Deeplex Myc-TB target on the total set of 1494 sputum samples and b) log read depth at Deeplex Myc-TB targets according to acid-fast bacilli (AFB) microscopy grading of 1143 sputum samples with available microscopic examination data. Box and whisker plots show median with interquartile range (IQR) and minimum-maximum range with a maximum of $1.5 \mathrm{IQR}$, respectively.

\section{Discussion}

Based on a large dataset, our results show a high degree of accuracy of the Deeplex Myc-TB assay for extensive prediction of both susceptibility and resistance to anti-tuberculous drugs with an efficiency close to WGS, directly achievable at least from AFB smear-positive clinical specimens. In contrast, susceptibility in particular cannot be reliably inferred from a negative result with conventional molecular tests, because of the limited set of resistance-conferring mutations covered [14].

Importantly, most of the residual predictions of Deeplex Myc-TB discordant with susceptible or resistant phenotypes in the tested set of 429 MTBC isolates involved pyrazinamide, ethambutol and ethionamide, for which pDST is an imperfect standard $[3,31]$. Likewise, for isoniazid or rifampicin, the sole discrepant resistant predictions by Deeplex Myc-TB all involved low-level resistance variants, frequently missed by liquid pDST, although they are critical to capture to avoid unfavourable treatment outcome or relapse [3, 32, 33]. For these reasons, sequencing of relevant genes is now proposed as a reference, at least for rifampicin, pyrazinamide, ethambutol and ethionamide [31]. Deeplex Myc-TB can thus be considered as outperforming the pDST standard for these drugs.

Crucially in this respect, the agreement with WGS analysis was almost complete in both the in silico and experimental datasets. All heteroresistant calls detected by both methods in the set of 429 MTBC strains were concordant with resistance phenotypes. The sole heteroresistant variant exclusively detected (at $4.9 \%$ ), in an ethambutol-susceptible phenotype, by deeper sequencing with Deeplex Myc-TB involved an embB M306V variant. Variants in this embB 306 codon (as well as codons 354, 406 and 497) are assumed to be resistance mutations, regardless of the pDST result obtained [36]. Likewise, most of the resistance predictions made only by direct Deeplex Myc-TB analysis on sputa from the Djibouti survey were due to resistance minority variants, undetected by MTBSeq/PhyResSE/Mykrobe in WGS data likely due to insufficient read depths and/or culture bias eliminating potentially less fit (resistant) subpopulations [37] before subjection to WGS.

The optimal read depth-dependent limit of 3\% for detecting minority populations with this Deeplex Myc-TB version was defined to secure true variant calls, after systematic analysis of sequence noise levels across target positions. This limit is substantially better than those of conventional molecular tests [38], although not as sensitive as the $0.1-1 \%$ limit claimed for the TGen deep-sequencing assay [10, 39]. While we recently developed an enhanced detection mode capable of reliably detecting minority variants down to $1 \%$, this can only be reached on hypercovered target portions. Going below this threshold results in an 
unacceptably higher rate of false-positive base calls, affecting the overall specificity of such assays (data not shown). A genetic cut-off around this value seems reasonable, as old studies using the phenotypic proportion method showed that therapeutic success was unlikely above $1 \%$ of growing drug-resistant bacilli [40]. However, the correspondence between phenotypic and genetic estimates could be influenced by the uncertainty of phenotypic proportions given the clumping nature of mycobacteria and/or fitness cost of some resistance mutations resulting in underrepresentation of the cultured resistant subpopulation [37]. More studies are needed to address this important question.

Despite its large dataset, this study has limitations. The reference collection of 429 strains included relatively few resistant phenotypes for the second-line drugs investigated (none for bedaquiline and linezolid). Nevertheless, as also partly suggested from our in silico analysis, the Deeplex Myc-TB targets and mutation catalogue comprise the main or even the exclusively established genomic targets and most determinants of resistance to these drugs in clinical isolates (e.g. gyrA and gyrB quinolone resistance-determining regions for the fluoroquinolones). pDST data were not available for clinical specimens from Djibouti and the DRC, as pDST is not routinely performed on all newly diagnosed TB patients in such resource-limited countries, and because of the difficulty to re-culture samples even with preservatives added upon storage and transportation. However, our results showed closely matching phenotype predictions between Deeplex Myc-TB and the ultimate genotypic reference, i.e. WGS analysis, on the Djibouti dataset. Finally, the number of tested smear-negative samples clearly identified as such in the DRC dataset was limited. Nevertheless, the established limit of detection of 100-1000 extracted genome copies indicates that the test can be applied on all smear-positive samples and at least part of smear-negative samples with any reasonably efficient DNA extraction method. Consistently, the similar mean read depths obtained on the specimens graded $1+, 2+$ and $3+$ from the DRC survey also suggest a limit of detection $\leqslant 1+$ (corresponding to $5000-10000$ genomes $\cdot \mathrm{mL}^{-1}$ of sample) with the DNA extraction conditions used, accounting also for the fact that the equivalent of about a tenth of the available $1-5 \mathrm{~mL}$ of the DRC samples was generally used for DNA extraction and testing. Further consistent with such analytical sensitivity, in a recent study using QIAamp DNA Mini kits for DNA extraction, complete Deeplex Myc-TB phenotypic predictions could be made from all 37 smear-positive (including five scanty) and two smear-negative samples, in a pilot series of 50 clinical specimens [41].

In conclusion, the results of this extensive evaluation demonstrate the potential of the Deeplex Myc-TB assay to reliably guide personalised $\mathrm{TB}$ treatment, from culture or directly from clinical specimens depending on their bacterial loads. As the test can also be used on Illumina iSeq100 and MiniSeq, in addition to the MiSeq and NextSeq platforms used here, the scalability of throughputs, with optimal batches of 16 (iSeq100) to 384 tests per run (NextSeq) (including three controls per run), can cover the needs of many clinical laboratories at local/regional or nationally centralised levels. Its use might be particularly cost-effective upon positive MTBC detection (with or without rifampicin resistance) with a more sensitive rapid triage test such as Xpert MTB/RIF. Particularly when used on sputum, this assay will significantly reduce the total turnaround for generating extended DST reports. Culturing could potentially be restricted to samples without conclusive Deeplex Myc-TB results and pDST potentially limited to drugs for which uncharacterised mutations (instead of resistance, benign or no mutation) are detected in the relevant genes, as proposed for WGS-based phenotype predictions from culture [3], or for the new/ repurposed drugs bedaquiline, clofazimine and delamanid/pretomanid, for which resistance mutations are poorly characterised ( $R v 0678$ for bedaquiline/clofazimine) or not (yet) covered by the assay (atpE and $p e p Q$ for bedaquiline and all (candidate) genes for delamanid and pretomanid). Planned improvements include addition of such extra targets.

Acknowledgements: We thank Mathilde Mairey (GenoScreen, Lille, France) for valuable technical help.

Sequencing data have deposited in the National Center for Biotechnology Information Sequence Read Archive, with BioProject numbers provided. Detailed analysis data are provided in supplementary tables S1-S13.

Support statement: Parts of this work have been supported by the European Union PathoNGen-Trace project (FP7-278864) and by the World Health Organization (for the TB drug resistance survey conducted in the Democratic Republic of the Congo). B.C. de Jong and L. Rigouts were supported by European Research Council starting grant "INTERRUPTB" (grant agreement 311725). Funding information for this article has been deposited with the Crossref Funder Registry.

Conflict of interest: A. Jouet is an employee of GenoScreen. C. Gaudin is an employee of GenoScreen. N. Badalato is an employee of GenoScreen. C. Allix-Béguec is an employee of GenoScreen. S. Duthoy is an employee of GenoScreen. A. Ferré is an employee of GenoScreen. M. Diels has nothing to disclose. Y. Laurent is an employee of GenoScreen. S. Contreras is an employee of GenoScreen. S. Feuerriegel has nothing to disclose. S. Niemann reports grants from the German Center for Infection Research, Excellenz Cluster Precision Medicine in Chronic Inflammation EXC 2167, Leibniz Science Campus Evolutionary Medicine of the LUNG (EvoLUNG) and ECDC public tender: OJ/2017/OCS/7766

"Pilot study on the use of Whole Genome Sequencing for molecular typing and characterisation of M. tuberculosis in 
the EU/EEA", during the conduct of the study. E. André has nothing to disclose. M.K. Kaswa has nothing to disclose. E. Tagliani has nothing to disclose. A. Cabibbe has nothing to disclose. V. Mathys has nothing to disclose. D. Cirillo has nothing to disclose. B.C. de Jong has nothing to disclose. L. Rigouts has nothing to disclose. P. Supply reports personal fees for consultancy from GenoScreen, grants from the European Union PathoNGen-Trace project (FP7-278864), during the conduct of the study.

\section{References}

World Health Organization. Global Tuberculosis Report. Geneva, WHO, 2019.

2 Cabibbe AM, Walker TM, Niemann S, et al. Whole genome sequencing of Mycobacterium tuberculosis. Eur Respir J 2018; 52: 1801163 .

3 Walker TM, Kohl TA, Omar SV, et al. Whole-genome sequencing for prediction of Mycobacterium tuberculosis drug susceptibility and resistance: a retrospective cohort study. Lancet Infect Dis 2015; 15: 1193-1202.

4 The CRyPTIC Consortium and the 100,000 Genomes Project. Prediction of susceptibility to first-line tuberculosis drugs by DNA sequencing. N Engl J Med 2018; 379: 1403-1415.

5 Zignol M, Cabibbe AM, Dean AS, et al. Genetic sequencing for surveillance of drug resistance in tuberculosis in highly endemic countries: a multi-country population-based surveillance study. Lancet Infect Dis 2018; 18: 675-683.

6 Pankhurst LJ, Del Ojo Elias C, Votintseva AA, et al. Rapid, comprehensive, and affordable mycobacterial diagnosis with whole-genome sequencing: a prospective study. Lancet Respir Med 2016; 4: 49-58.

7 Doyle RM, Burgess C, Williams R, et al. Direct whole-genome sequencing of sputum accurately identifies drug-resistant Mycobacterium tuberculosis faster than MGIT culture sequencing. J Clin Microbiol 2018; 56: e00666-18.

8 George S, Xu Y, Rodger G, et al. DNA thermo-protection facilitates whole genome sequencing of mycobacteria direct from clinical samples by the Nanopore platform. BioRxiv 2020; preprint [https://doi.org/10.1101/2020.04.05. 026864].

9 Vargas R, Freschi L, Marin M, et al. In-host population dynamics of $M$. tuberculosis during treatment failure. BioRxiv 2020; preprint [https://doi.org/10.1101/726430].

10 Colman RE, Schupp JM, Hicks ND, et al. Detection of low-level mixed-population drug resistance in Mycobacterium tuberculosis using high fidelity amplicon sequencing. PLoS One 2015; 10: e0126626.

11 Colman RE, Mace A, Seifert M, et al. Whole-genome and targeted sequencing of drug-resistant Mycobacterium tuberculosis on the iSeq100 and MiSeq: a performance, ease-of-use, and cost evaluation. PLoS Med 2019; 16 e1002794.

12 Colman RE, Anderson J, Lemmer D, et al. Rapid drug susceptibility testing of drug-resistant Mycobacterium tuberculosis isolates directly from clinical samples by use of amplicon sequencing: a proof-of-concept study. J Clin Microbiol 2016; 54: 2058-2067.

13 Tagliani E, Hassan MO, Waberi Y, et al. Culture and next-generation sequencing-based drug susceptibility testing unveil high levels of drug-resistant-TB in Djibouti: results from the first national survey. Sci Rep 2017; 7: 17672.

14 Makhado NA, Matabane E, Faccin M, et al. Outbreak of multidrug-resistant tuberculosis in South Africa undetected by WHO-endorsed commercial tests: an observational study. Lancet Infect Dis 2018; 18: 1350-1359.

15 El Achkar S, Demanche C, Osman M, et al. Zoonotic tuberculosis in humans assessed by next-generation sequencing: an 18-month nationwide study in Lebanon. Eur Respir J 2020; 55: 1900513.

16 Vincent V, Rigouts L, Nduwamahoro E, et al. The TDR Tuberculosis Strain Bank: a resource for basic science, tool development and diagnostic services. Int J Tuberc Lung Dis 2012; 16: 24-31.

17 Pfyffer GE, Bonato DA, Ebrahimzadeh A, et al. Multicenter laboratory validation of susceptibility testing of Mycobacterium tuberculosis against classical second-line and newer antimicrobial drugs by using the radiometric BACTEC 460 technique and the proportion method with solid media. J Clin Microbiol 1999; 37: 3179-3186.

18 Cambau E, Viveiros M, Machado D, et al. Revisiting susceptibility testing in MDR-TB by a standardized quantitative phenotypic assessment in a European multicentre study. J Antimicrob Chemother 2015; 70: 686-696.

19 Kohl TA, Utpatel C, Schleusener V, et al. MTBseq: a comprehensive pipeline for whole genome sequence analysis of Mycobacterium tuberculosis complex isolates. PeerJ 2018; 2018; 6: e5895.

20 Feuerriegel S, Schleusener V, Beckert P, et al. PhyResSE: a web tool delineating Mycobacterium tuberculosis antibiotic resistance and lineage from whole-genome sequencing data. J Clin Microbiol 2015; 53: 1908-1914.

21 Bradley P, Gordon NC, Walker TM, et al. Rapid antibiotic-resistance predictions from genome sequence data for Staphylococcus aureus and Mycobacterium tuberculosis. Nat Commun 2015; 6: 10063.

22 Kayomo MK, Mbula VN, Aloni M, et al. Targeted next-generation sequencing of sputum for diagnosis of drug-resistant TB: results of a national survey in Democratic Republic of the Congo. Sci Rep 2020; 10: 10786.

23 Dai J, Chen Y, Lauzardo M. Web-accessible database of $h s p 65$ sequences from Mycobacterium reference strains. J Clin Microbiol 2011; 49: 2296-2303.

24 Demay C, Liens B, Burguière T, et al. SITVITWEB - a publicly available international multimarker database for studying Mycobacterium tuberculosis genetic diversity and molecular epidemiology. Infect Genet Evol 2012; 12 755-766.

25 Coll F, McNerney R, Guerra-Assunção JA, et al. A robust SNP barcode for typing Mycobacterium tuberculosis complex strains. Nat Commun 2014; 5: 4812.

26 Miotto P, Cabibbe AM, Feuerriegel S, et al. Mycobacterium tuberculosis pyrazinamide resistance determinants: a multicenter study. mBio 2014; 5: e01819-14.

27 Miotto P, Tessema B, Tagliani E, et al. A standardised method for interpreting the association between mutations and phenotypic drug resistance in Mycobacterium tuberculosis. Eur Respir J 2017; 50: 1701354.

28 Meehan CJ, Goig GA, Kohl TA, et al. Whole genome sequencing of Mycobacterium tuberculosis: current standards and open issues. Nat Rev Microbiol 2019; 17: 533-545.

29 Yadon AN, Maharaj K, Adamson JH, et al. A comprehensive characterization of PncA polymorphisms that confer resistance to pyrazinamide. Nat Commun 2017; 8: 588

30 Baulard AR, Betts JC, Engohang-Ndong J, et al. Activation of the pro-drug ethionamide is regulated in mycobacteria. J Biol Chem 2000; 275: 28326-28331. 
31 World Health Organization. Technical Manual for Drug Susceptibility Testing of Medicines Used in the Treatment of Tuberculosis. Geneva, WHO, 2018.

32 Miotto P, Cabibbe AM, Borroni E, et al. Role of disputed mutations in the rpoB gene in interpretation of automated liquid MGIT culture results for rifampin susceptibility testing of Mycobacterium tuberculosis. J Clin Microbiol 2018; 56: e01599-1.

33 Lempens P, Meehan CJ, Vandelannoote K, et al. Isoniazid resistance levels of Mycobacterium tuberculosis can largely be predicted by high-confidence resistance-conferring mutations. Sci Rep 2018; 8: 3246.

34 Manson AL, Cohen KA, Abeel T, et al. Genomic analysis of globally diverse Mycobacterium tuberculosis strains provides insights into the emergence and spread of multidrug resistance. Nat Genet 2017; 49: 395-402.

35 Supply P, Marceau M, Mangenot S, et al. Genomic analysis of smooth tubercle bacilli provides insights into ancestry and pathoadaptation of Mycobacterium tuberculosis. Nat Genet 2013; 45: 172-179.

36 Andres S, Gröschel MI, Hillemann D, et al. A diagnostic algorithm to investigate pyrazinamide and ethambutol resistance in rifampin-resistant Mycobacterium tuberculosis isolates in a low-incidence setting. Antimicrob Agents Chemother 2019; 63: e01798-18. .

37 Gagneux S, Long CD, Small PM, et al. The competitive cost of antibiotic resistance in Mycobacterium tuberculosis. Science 2006; 312: 1944-1946.

$38 \mathrm{Ng}$ KCS, Supply P, Cobelens FGJ, et al. How well do routine molecular diagnostics detect rifampin heteroresistance in Mycobacterium tuberculosis? J Clin Microbiol 2019; 57: e00717-19.

39 Metcalfe JZ, Streicher E, Theron G, et al. Cryptic microheteroresistance explains Mycobacterium tuberculosis phenotypic resistance. Am J Respir Crit Care Med 2017; 196: 1191-1201.

40 Canetti G, Fox W, Khomenko A, et al. Advances in techniques of testing mycobacterial drug sensitivity, and the use of sensitivity tests in tuberculosis control programmes. Bull World Health Organ 1969; 41: 21-43.

41 Feuerriegel S, Kohl TA, Utpatel C, et al. Rapid genomic first- and second-line drug resistance prediction from clinical tuberculosis specimens using Deeplex Myc-TB. Eur Respir J 2020; 57: 2001796. 